\title{
Indentation of power law creep solids by self-similar indenters
}

\author{
Wei-Min Chen ${ }^{a}$, Yang-Tse Cheng ${ }^{b}$, Min Lic,* \\ a Laboratory of Environmental Mechanics, Institute of Mechanics, Chinese Academy of Sciences, Beijing 100190, China \\ ${ }^{\mathrm{b}}$ Department of Chemical and Materials Engineering, University of Kentucky, Lexington, KY 40506-0046, USA \\ c School of Aeronautics Sciences and Technology, Beijing University of Aeronautics and Astronautics, Beijing 100191, China
}

\section{A R T I C L E I N F O}

\section{Article history:}

Received 30 November 2009

Received in revised form 5 May 2010

Accepted 23 May 2010

\section{Keywords:}

Indentation

Power law creep

Dimensional analysis

Self-similar indenters

\section{A B S T R A C T}

For creep solids obeying the power law under tension proposed by Tabor, namely

$\sigma=b \dot{\varepsilon}^{m}$

it has been established through dimensional analysis that for self-similar indenters the load $F$ versus indentation depth $h$ can be expressed as

$F(t)=b h^{2}(t)\left[\frac{\dot{h}(t)}{h(t)}\right]^{m} \Pi_{\alpha}$

where the dimensionless factor $\Pi_{\alpha}$ depends on material parameters such as $m$ and the indenter geometry. In this article, we show that by generalizing the Tabor power law to the general three dimensional case on the basis of isotropy, this factor can be calculated so that indentation test can be used to determine the material parameters $b$ and $m$ appearing in the original power law. Hence indentation test can replace tension test. This could be a distinct advantage for materials that come in the form of thin films, coatings or otherwise available only in small amounts. To facilitate application values of this constant are given in tabulated form for a range of material parameters.

(C) 2010 Elsevier B.V. All rights reserved.

\section{Introduction}

The development of thin film technology, MEMS, nanotechnology, etc., places ever greater demand on the measurement of the mechanical properties of systems at micro to sub-micro scales. To meet this demand the nano-indentation technology has received considerable attention. Many of these small-scale materials, e.g. polymers, composites, biomaterials and food products, are "soft", or exhibit viscoelastic behavior such as creep. In fact theoretical studies of liner viscoelastic bodies in contact became active since the mid 1950 s by work of Lee [1], Radok [2], Lee and Radok [3], Hunter [4], Graham [5,6], Yang [7], and Ting [8,9]. In recent years, a number of authors have extended the early work to the analysis of indentation measurements in viscoelastic solids using either conical or spherical indenters [10-15]. Cheng et al. [16-18] investigated indentation in linear viscoelastic solids. Specifically, they examined the relationship between initial unloading slope, contact depth, and

\footnotetext{
* Corresponding author. Fax: +8610 82338527

E-mail address: limin@buaa.edu.cn (M. Li).
}

mechanical properties and investigated whether the commonly used Oliver-Pharr method for determining the contact depth or contact area is applicable to indentation in viscoelastic solids. Their works help improve the understanding of indentation in linear viscoelastic solids and resolve questions raised in recent literature about measuring viscoelastic properties from indentation experiments.

This paper addresses the problem of indentation of the creep solids by self-similar indenters. Aside from the spherical indenter which is not self-similar, such commonly used indenters as the conic, Vickers, Berkovich and Knoop indenters all belong to this class. A widely used empirical creep law under unidirectional tension proposed by Mulhearn and Tabor [19] in 1960 will be used as the basis for the present work. Based on this law much analytical work has been done. An important feature is that in many cases the creep problem can be reduced to the solution of a static nonlinear elasticity problem. The problem of indentation is one of such cases (Bower et al. [20]).

In this paper Tabor's creep law

$$
\sigma=b \dot{\varepsilon}^{m}
$$


where $\sigma$ and $\dot{\varepsilon}$ are the stress and strain rate respectively and $b$ and $m$ are material constants, is cast in the following generalized form

$$
\begin{aligned}
\sigma_{i j}= & \frac{b}{(1+\alpha)^{m}}\left[\left(\dot{\varepsilon}_{\ln }+\frac{\alpha}{1-2 \alpha} \delta_{\ln } \dot{\varepsilon}_{k k}\right)\left(\dot{\varepsilon}_{l m}+\frac{\alpha}{1-2 \alpha} \delta_{\ln } \dot{\varepsilon}_{k k}\right)\right]^{(m-1) / 2} \\
& \times\left(\dot{\varepsilon}_{i j}+\frac{\alpha}{1-2 \alpha} \delta_{i j} \dot{\varepsilon}_{k k}\right)
\end{aligned}
$$

where $\sigma_{i j}$ and $\dot{\varepsilon}_{\text {ln }}$ indicate the tensors of stress and strain rate respectively and the subscripts refer to Cartesian axes. In Eq. (2) an additional dimensionless material constant $\alpha$ is introduced to take into account of the lateral contraction of materials after the manner of the Poison's ratio in elasticity. Note that in both equations, only instantaneous strain rate appears. In this sense, such a material actually behaves like a fluid rather than a solid in that the constitutive relation does not depend on the instantaneous strain. Hence it is convenient to express the strain rate tensor $\dot{\varepsilon}_{i j}$ in Euler coordinate system in terms the velocity components $u_{i}$ as follows,

$\dot{\varepsilon}_{i j}=\frac{1}{2}\left(u_{i, j}+u_{j, i}\right)$

We denote the depth of indentation and the rate of penetration by $h(t)$ and $\dot{h}(t)$ respectively. For self-similar indenters, dimensional analysis [21] provides the following formulas for the indentation force $F$ and the projected area $A_{c}$ of the contact surface

$F(t)=b h^{2}(t)\left[\frac{\dot{h}(t)}{h(t)}\right]^{m} \Pi_{\alpha}(m, \alpha, \vartheta)$
$A_{c}(t)=h^{2}(t) \Pi_{\beta}(m, \alpha, \vartheta)$

where $\Pi_{\alpha}(m, \alpha, \vartheta)$ and $\Pi \beta(m, \alpha, \vartheta)$ are the functions of the exponent $m$, the lateral contraction coefficient $\alpha$ and $\vartheta$ representing a dimensionless characteristic number or a set of characteristic numbers defining the geometry of the indenter. It follows that the hardness is given by

$$
\begin{aligned}
H \equiv \frac{F}{A_{c}}= & b \Pi_{\gamma}(m, \alpha, \vartheta)\left(\frac{\dot{h}}{h}\right)^{m} \\
& \text { with } \Pi_{\gamma}(m, \alpha, \vartheta)=\frac{\Pi_{\alpha}(m, \alpha, \vartheta)}{\Pi_{\beta}(m, \alpha, \vartheta)}
\end{aligned}
$$

In the following section, we show how these functions can be calculated for given values of $b, m$ and $\alpha$. Then the problem of determining the three material constants, $b, m$ and $\alpha$ in Eq. (2) becomes an inverse problem which is solved by curve fitting of the experimental data through the knowledge of two of the three $\prod$ 's (the other follows from Eq. (4)). Note that so far the statement of the problem is not limited to small deformation or to small strain.

The standard Vickers indenter has an equilateral square base. The standard Berkovich has a triangular base. For them $\vartheta$ may be defined as the ratio of the base area to the square of the height of the pyramid, and its value is equal to 24.504 and 24.566 respectively. $\mathrm{Li}$ et al. [22] demonstrated numerically that for elastic-plastic materials (1) these two types of indenters yield nearly identical load and displacement curves ( $F$ versus $h$ ); (2) the conic indenter defined by identifying the parameter $\vartheta$ with the half apex angle equal to $70.3^{\circ}$ produces nearly indistinguishable $F \sim h$ relations. Hence the numerical part of the present study need only deal with the conic indenter.

\section{Formulation of the problem}

In terms of Cartesian tensors, the stress tensor $\sigma_{i j}$ satisfies the following equations of equilibrium when the Euler coordinate system is adopted

$\sigma_{i j, j}=0$
Eqs. (2), (3) and (6) form the fundamental set of equations for our problem. The stress $\sigma_{i j}$ and velocity $u_{i}$ must satisfy the following boundary conditions appropriate to the indentation problem. On the surface $A$ of the indenter in contact with the material being tested the normal velocity must be continuous and the shear stress is taken to be zero (no friction). On the free surface of the material not in contact with the indenter the surface stress must be zero. These two surfaces are delineated by the contour $\Gamma$. At infinity all stress components vanish.

These conditions can be made explicit mathematically in the following way. Let us denote the direction of the penetration velocity $\dot{h}$ by the unit vector $l_{i}$ and the unit normal vector and the two unit tangential vectors of $A$ by $n_{i}$ (directing toward the indenter) $\tau_{i}$ and $s_{i}$ respectively. Then for points inside $\Gamma$ (points in contact with the indenter), the following conditions must be satisfied,

$$
\begin{aligned}
& \left(u_{i}-\dot{h} l_{i}\right) n_{i}=0 \\
& \sigma_{i j} n_{i} \tau_{j}=0 \\
& \sigma_{i j} n_{i} s_{j}=0
\end{aligned}
$$

On the free surface (points outside $\Gamma$ ) where the unit normal vector is also denoted by $n_{i}$, it is required that

$\sigma_{i j} n_{i}=0$

At points away from the indenter

$\sigma_{i j} \rightarrow 0$

In addition to the boundary conditions the initial conditions are

At $t=0$

$h(0)=0$

$u_{i}=0$ through out the specimen

$\sigma_{i j}=0$ through out the specimen

so that the specimen is initially at rest, without displacement and stress free.

Finally, $F$ can be calculated from

$F=-\int_{A} \sigma_{i j} n_{i} l_{j} \mathrm{~d} S$

$\mathrm{d} S$ being an element of the contact surface $A$. The penetration depth $h(t)$ is obviously given by

$h(t)=\int_{0}^{t} \dot{h}(\tau) \mathrm{d} \tau$

The projected contact area $A_{c}$ is given by

$A_{C}=-\int_{A} n_{i} l_{i} \mathrm{~d} S$

\section{Similarity and reduction to a non-linear elasticity problem}

Reduction of the creep problem in indentation to a nonlinear elasticity problem has been discussed by Bower et al. (1993). For self-similar indenters the problem can be further simplified by observing that $h$ and $\dot{h}$ are the only measures of length and velocity respectively. Thus by dimensional argument we can introduce the dimensionless space coordinates $\xi_{i}$ and dimensionless "displacement" $v_{i}$ by

$$
\begin{aligned}
& x_{i}=h \xi_{i} \\
& u_{i}=\dot{h} v_{i}
\end{aligned}
$$

In terms of this new independent coordinate system $\xi_{i}$ and $v_{i}$, we have

$\dot{\varepsilon}_{i j}=\frac{1}{2} \frac{\dot{h}}{h}\left(v_{i, j}+v_{j, i}\right)$ 


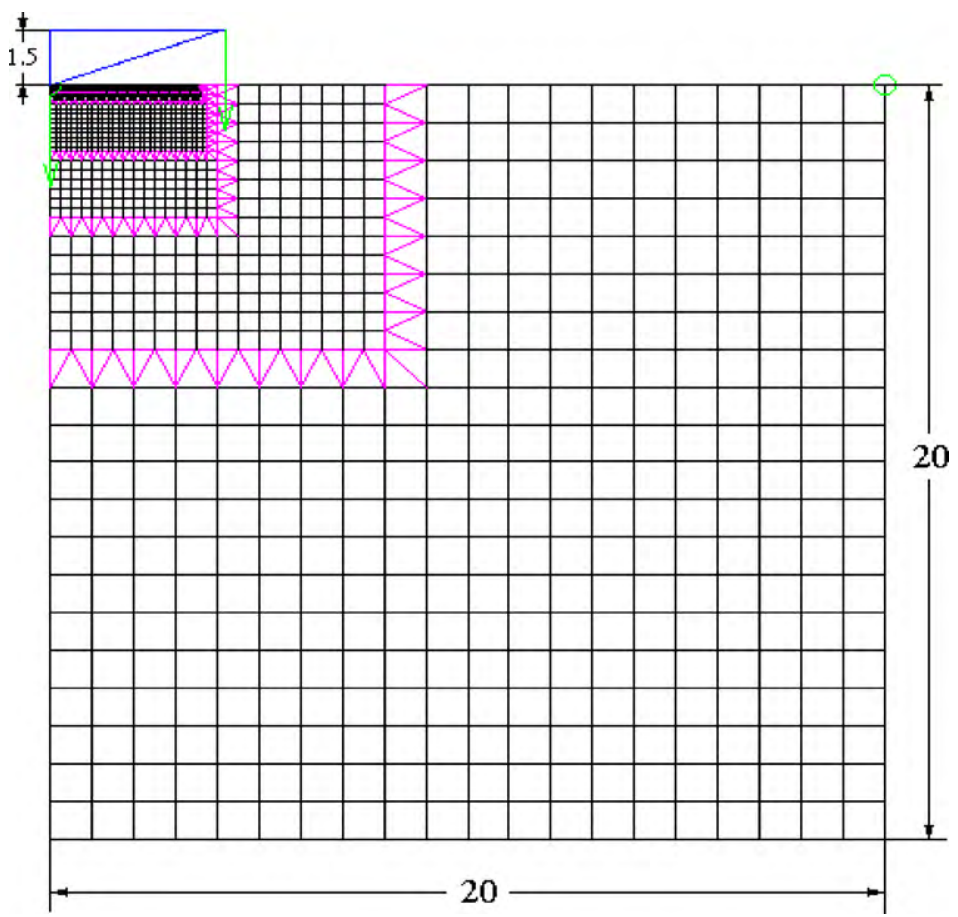

Fig. 1. A typical mesh for finite element modeling.

Following this relation we may define a strain tensor $e_{i j}$ by

$e_{i j}=\frac{1}{2}\left(v_{i, j}+v_{j, i}\right)$

In accordance with the creep law Eq. (2) we can define a new stress tensor $s_{i j}$ by

$$
\begin{aligned}
s_{i j}= & \frac{1}{(1+\alpha)^{m}}\left[\left(e_{\ln }+\frac{\alpha}{1-2 \alpha} \delta_{\ln } e_{k k}\right)\left(e_{\ln }+\frac{\alpha}{1-2 \alpha} \delta_{\ln } e_{k k}\right)\right]^{(m-1) / 2} \\
& \times\left(e_{i j}+\frac{\alpha}{1-2 \alpha} \delta_{i j} e_{k k}\right)
\end{aligned}
$$

so that

$\sigma_{i j}=b\left(\frac{\dot{h}}{h}\right)^{m} s_{i j}$

Consequently the corresponding "equations of equilibrium" are

$s_{i j, j}=0$

in terms of $\xi_{i}$. Eqs. (16), (17) and (19) now form a complete set of equations in nonlinear elasticity. They are to be solved under the following boundary conditions, namely on the dimensionless contact surface $A^{\prime}$ ( $A$ scaled by $h^{2}$ ),

$v_{3}=1, v_{1}=v_{2}=0$ at the tip of the indenter

$\left(v_{i}-l_{i}\right) n_{i}=0$

$s_{i j} n_{i} \tau_{j}=0$

$s_{i j} n_{i} s_{j}=0$

while on the free surface

$s_{i j} n_{i}=0$

Also, $s_{i j} \rightarrow 0$ at points far away from the indenter. The initial configuration of the specimen is a semi-infinite plane and deformation, strain and stress field for this fictitious nonlinear elastic problem is to be calculated for a penetration depth of unity. According to the transformation rule Eq. (14) the indenter moves with unit velocity.
But velocity is of no relevance in the solution of the static nonlinear elastic problem because inertia forces do not play a role in the present creep problem.

Another remark is called for. In the formulation of nonlinear elasticity and in terms of the Euler coordinate system the expression for the strain tensor is

$e_{i j}=\frac{1}{2}\left(v_{i, j}+v_{j, i}+v_{h, i} v_{h, j}\right)$

Comparing Eqs. (16) and (22) we conclude that in the reduction of the creep problem to a nonlinear elasticity problem the nonlinear terms in Eq. (22) may be neglected.

Making the same transformation in Eqs. (11) and (13) and referring to the definition of the nondimensional $\prod$ 's we obtain

$\Pi_{\alpha}(m, \alpha, \vartheta)=-\int_{A^{\prime}} s_{i j} n_{i} l_{j} \mathrm{~d} S^{\prime}$

$\Pi_{\beta}(m, \alpha, \vartheta)=-\int_{A^{\prime}} n_{i} l_{i} \mathrm{~d} S^{\prime}$

$\Pi_{\gamma}(m, \alpha, \vartheta)=\frac{\int_{A^{\prime}} s_{i j} n_{i} l_{j} \mathrm{~d} S^{\prime}}{\int_{A^{\prime}} n_{i} l_{i} \mathrm{~d} S^{\prime}}$

Hence the problem of the indentation of a creep material by self-similar indenters is now reduced to the solution of the indentation of a nonlinear elastic problem for an indentation depth of unity. Clearly $\prod_{\alpha}$ can be identified with the load, $\prod_{\beta}$ with the projected contact area and $\prod \gamma$ with the hardness of the fictitious elastic material.

Invoking self-similarity once more it can be easily shown that if the tip displacement is $\kappa$ in stead of unity, then the corresponding $\Pi$ 's denoted by $\Pi_{\alpha}^{s}, \Pi_{\beta}^{s}$ and $\Pi_{\gamma}^{s}$ are related to their values at $\kappa=1$ by

$\Pi_{\alpha}^{s}=\kappa^{2} \Pi_{\alpha}$

$\Pi_{\beta}^{s}=\kappa^{2} \Pi_{\beta}$

$\Pi_{\gamma}^{s}=\Pi_{\gamma}$ 


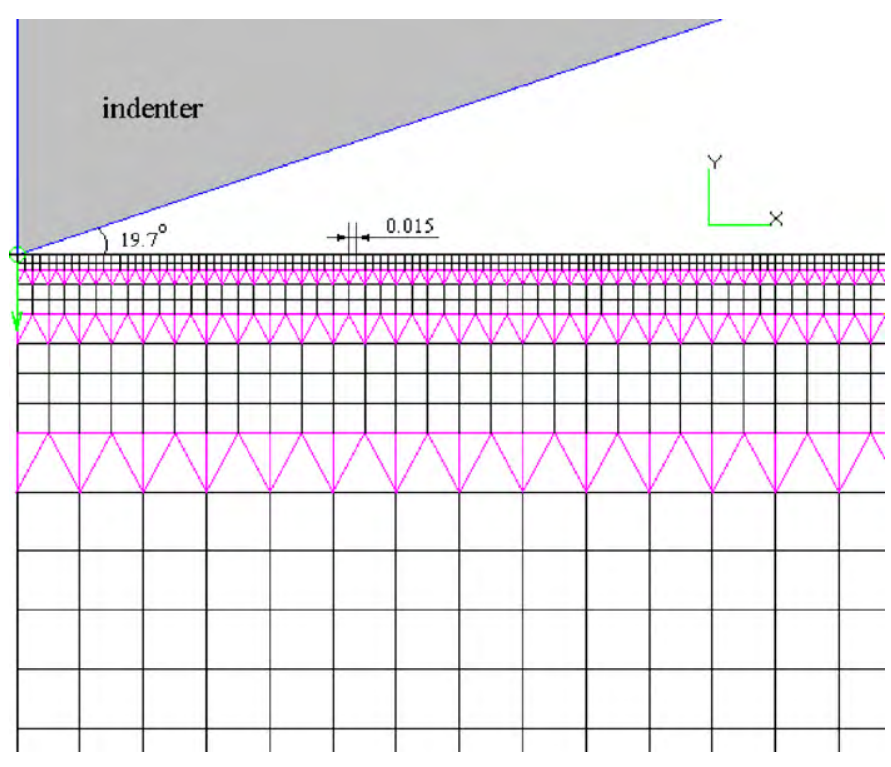

Fig. 2. The mesh in the vicinity of the indenter.

These relations are useful because in the actual execution of the numerical simulation, one has to first set up a grid which introduces an artificial length scale causing a violation of self-similarity at small indentation depth. The scale effect needs to be removed. Also one calculates the $\prod$ 's by gradually increasing the indentation depth in small steps up to $\kappa$. $\prod_{\alpha}, \prod_{\beta}$ and $\prod_{\gamma}$ as well as the power of $\kappa$ can then be obtained by regression.

\section{The numerical scheme and results}

\subsection{The numerical scheme}

Finite-element calculations using ABAQUS were carried out to obtain the values of $\Pi_{\alpha}^{s}, \Pi_{\beta}^{s}$ and $\Pi_{\gamma}^{s}$. Instead of the nonlinear model as prescribed by Eq. (17) a large strain elasto-plastic model satisfying Eq. (17) in loading is adopted, because no nonlinear elastic model is available in ABAQUS. To show that this substitution is valid our calculations were checked carefully against unloading and to our satisfaction no apparent unloading was found as long as $\dot{h}(t)$ does not change sign.

In the finite-element model shown in Fig. 1, the indenter and the nonlinear elastic solid were modeled as bodies of revolution to take advantage of the axisymmetry of conical indentation. The indenter was regarded as rigid with a half apex angle of $70.3^{\circ}$. The semiinfinite elastic-plastic solid was modeled using 3200 axisymmetric elements. In order to get accurate results close to the sharp tip of the indenter and for small indentation depth, a very fine mesh along the surface of the solid was made. For a maximum indentation depth equal to 1.0 the width of the elements along the contact surface is taken to be 0.015 (see Fig. 2).

Frictionless boundary conditions were applied along the axis of symmetry and on the contact surface. The free surface is stressless. The rest of the surface of the finite specimen is regarded as fixed. For sufficiently large specimen the last boundary condition does not affect the calculation.

The specimen is modeled as an elastic-plastic solid whose material property is given by the uniaxial stress-strain curve as required by Eq. (17). The index $m$ is taken to be $0.1,0.2,0.3,0.4,0.5$ and 1.0 , and the lateral contraction coefficient $\alpha$ is taken to be $0.100,0.250$, $0.300,0.450,0.490$ and 0.499 . A group of typical stress-strain curves for different values of $m$ is shown in Fig. 3 where $\alpha=0.300$. The nonlinear quasi-static calculation was carried out using ABAQUS

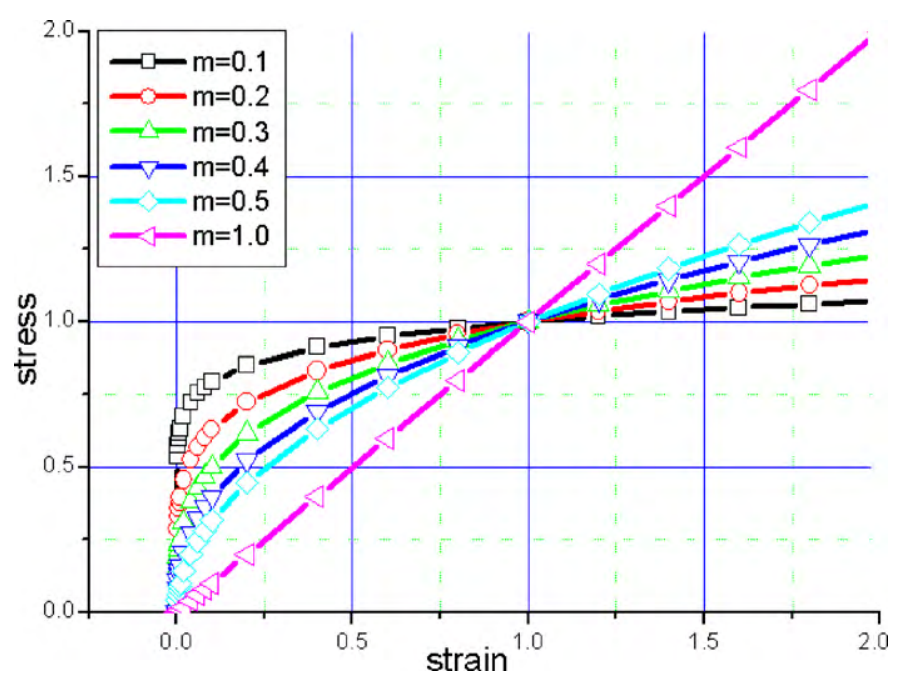

Fig. 3. The sketch of stress-strain by Eq. (17) $(\alpha=0.300)$.

Table 1

The regression parameters, $a_{r}$ and $b_{r}$ in $\Pi_{\alpha}=a_{r} \kappa^{b_{r}}$, from curves in Fig. 5a.

\begin{tabular}{llll}
\hline$m$ & $a_{r}$ & $b_{r}$ & $\begin{array}{l}\text { Correlation } \\
\text { coefficient } R^{2}\end{array}$ \\
\hline 0.1 & $70.319 \pm 0.236$ & $2.007 \pm 0.010$ & 0.9995 \\
0.2 & $46.977 \pm 0.145$ & $1.981 \pm 0.010$ & 0.9997 \\
0.3 & $32.768 \pm 0.054$ & $2.017 \pm 0.006$ & 0.9999 \\
0.4 & $22.254 \pm 0.031$ & $1.999 \pm 0.005$ & 0.9999 \\
0.5 & $15.389 \pm 0.007$ & $2.020 \pm 0.002$ & 1.0000 \\
1.0 & $2.210 \pm 0.001$ & $2.047 \pm 0.002$ & 1.0000 \\
\hline
\end{tabular}

by applying the indentation depth and then gradually increasing depth step by step.

\subsection{Numerical results}

Typical results of indentation process by finite-element calculation, the load-displacement curves as plot of $\Pi_{\alpha}^{s}$ versus $\kappa$ and the hardness-displacement curves as plot of $\Pi_{\gamma}^{s}$ versus $\kappa$, are shown in Fig. 4 (where $m$ ranges from 0.1 to 1.0 and $\alpha=0.300$ ). Eq. (24) indicates that both $\Pi_{\alpha}^{s}$ and $\Pi_{\beta}^{s}$ should be proportional to $\kappa^{2}$ and $\Pi_{\gamma}^{s}$ be independent of $\kappa$. The regression parameters, $a_{r}$ and $b_{r}$ in $\Pi_{\alpha}^{s}=a_{r} \kappa^{b_{r}}$ from the load-displacement curves in Fig. 4a, are given in Table 1. From Table 1 a goodness of fit is seen on fact that the values of correlation coefficient $R$ are nearly 1.0. It is also seen that the values of $b_{r}$ are almost consistent to the theoretical value, 2.0, required by dimensional analysis. Fig. $4 \mathrm{~b}$ shows that except at very small indentation depth $(\kappa<0.1)$ where mesh size introduces an artificial size effect, $\Pi_{\gamma}^{s}$ is independent of $\kappa$. Above results indicate that our calculations agree well with dimensional analysis. Additionally, in later section the values of $\prod_{\alpha}, \prod_{\beta}$ and $\prod_{\gamma}$ are given by smoothing the calculated curves, except points at very small indentation depth, as lines in Fig. 4b.

Table 2

$\prod_{\alpha}(m=0.1-1.0, \alpha=0.100-0.499)$.

\begin{tabular}{lllllll}
\hline$\alpha$ & $m=0.10$ & $m=0.20$ & $m=0.30$ & $m=0.40$ & $m=0.50$ & $m=1.00$ \\
\hline 0.100 & 68.162 & 45.986 & 31.119 & 20.973 & 14.518 & 2.089 \\
0.250 & 69.123 & 46.016 & 31.481 & 21.466 & 14.908 & 2.125 \\
0.300 & 70.203 & 47.165 & 32.668 & 22.258 & 15.343 & 2.196 \\
0.450 & 71.517 & 48.407 & 33.551 & 22.819 & 15.815 & 2.334 \\
0.490 & 72.995 & 49.213 & 33.811 & 23.399 & 16.180 & 2.426 \\
0.499 & 73.577 & 50.054 & 34.390 & 23.636 & 16.405 & 2.514 \\
\hline
\end{tabular}




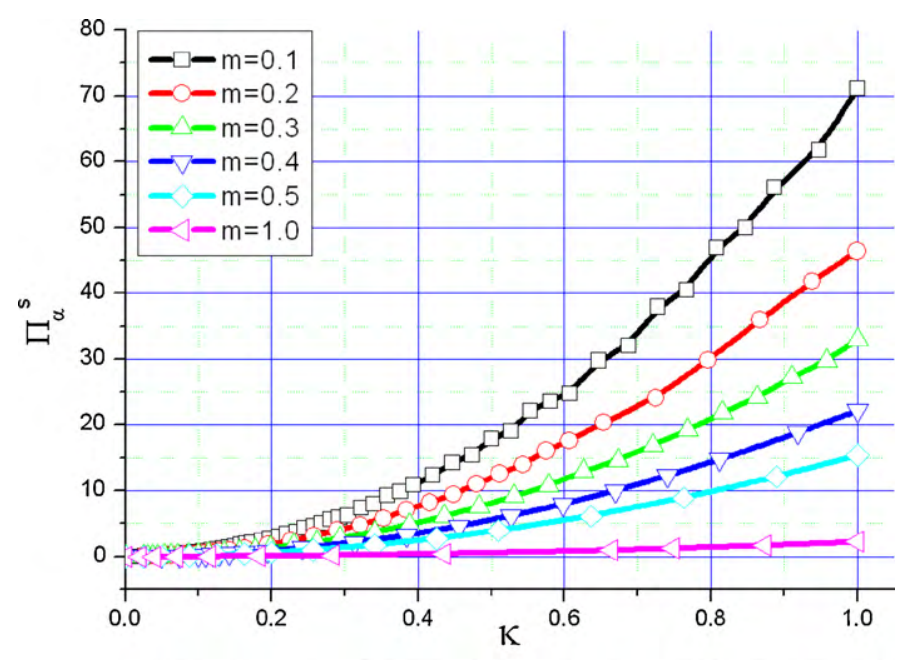

(a) The $\Pi_{\alpha}^{s}-\kappa$ curves ( or the load-displacement curves)

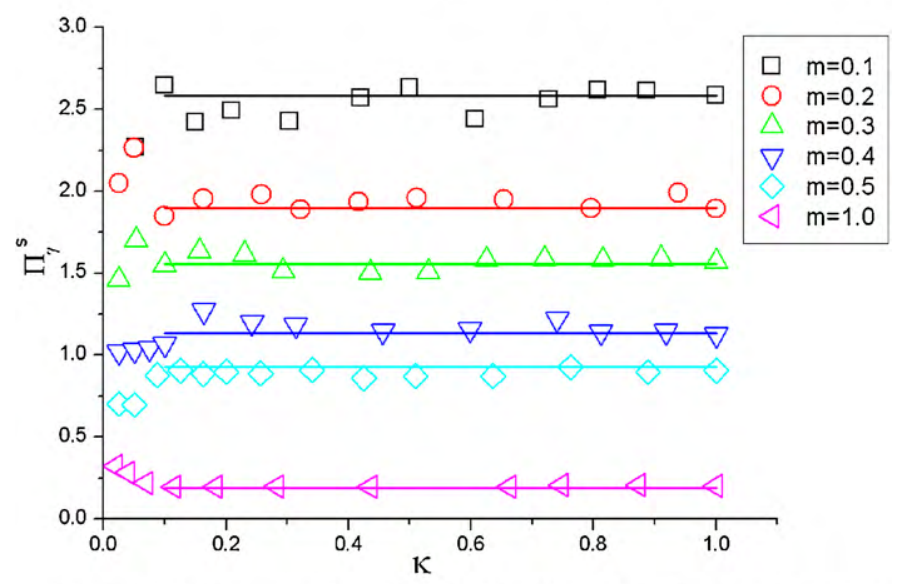

(b) The $\Pi_{\gamma}^{s}-\kappa$ curves ( or the hardness-displacement curves)

Fig. 4. Typical results of finite element calculation for $m=0.1-1.0$ and $\alpha=0.300$.

The values of the three $\prod$ 's for a rigid conic indenter with a half apex angle $70.3^{\circ}$ are given respectively in Tables $2-4$ for material parameters $m$ ranging from 0.1 to 1.0 and $\alpha$ from 0.100 to 0.499 . From Tables 2-4, it is seen that the values of three $\prod$ 's drop as the values of index $m$ increase, but almost remain constant (increase a few) as the values of $\alpha$ increase.

The influence of $\alpha$, not appearing in the original problem (Eq. (1)) but as an additional material constant introduced to take into account of the lateral contraction of materials after the manner of the Poison's ratio in elasticity, on calculation results is also observed here. The selected results, influences of $\alpha$ on values of $\prod \gamma$ and $b_{r}$ are shown in Fig. 5. Plot of $\prod \gamma$ against $\alpha$ with $m=0.1-1.0$ (Fig. 5a) shows that the value of $\prod_{\gamma}$ is nearly independent of values of $\alpha$ except as $\alpha$ approaching 0.5 and $m \leq 0.1$. This implies that the calculation results are not very sensitive to values of $\alpha$.

Table 3
$\prod_{\beta}(m=0.1-1.0, \alpha=0.100-0.499)$.

\begin{tabular}{lllllll}
\hline$\alpha$ & $m=0.10$ & $m=0.20$ & $m=0.30$ & $m=0.40$ & $m=0.50$ & $m=1.00$ \\
\hline 0.100 & 26.749 & 24.505 & 20.984 & 19.981 & 16.513 & 11.176 \\
0.250 & 26.749 & 24.505 & 20.984 & 19.981 & 16.513 & 11.284 \\
0.300 & 27.132 & 24.837 & 20.998 & 19.678 & 16.513 & 11.472 \\
0.450 & 27.518 & 25.242 & 20.984 & 20.313 & 16.513 & 11.683 \\
0.490 & 27.518 & 25.242 & 21.324 & 20.647 & 17.119 & 12.089 \\
0.499 & 27.518 & 25.242 & 21.324 & 20.647 & 17.119 & 12.089 \\
\hline
\end{tabular}

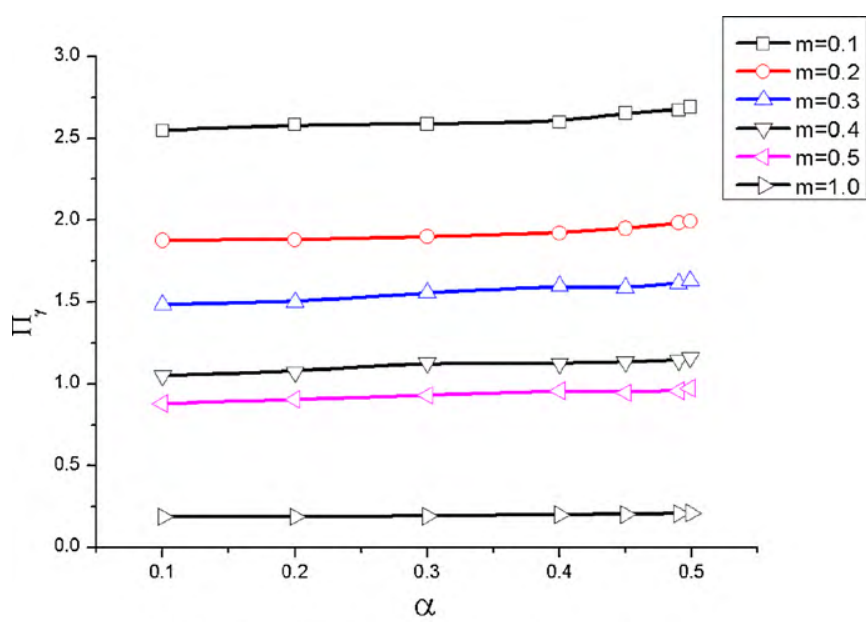

(a) $\Pi_{\gamma}$ versus $\alpha$ for $m=0.1 \sim 1.0$

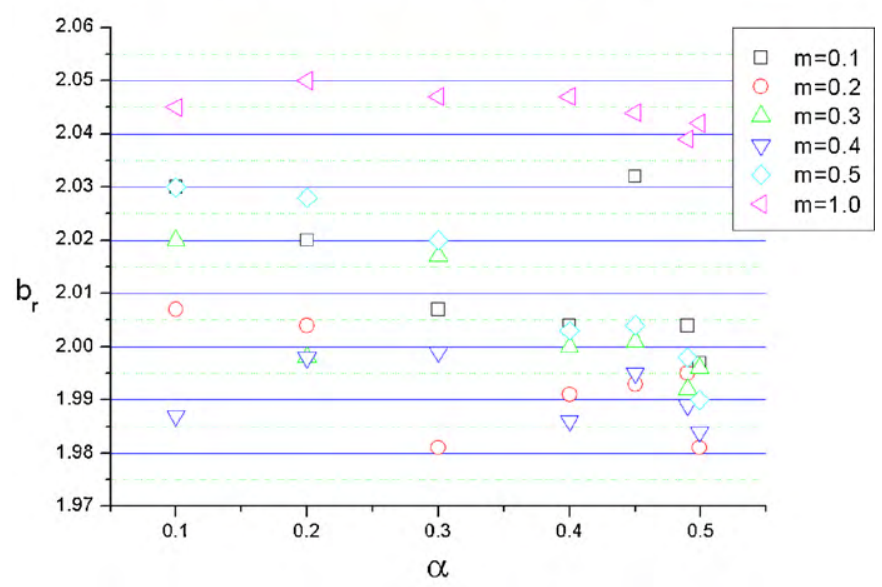

(b) Regression parameter $b_{r}$ versus $\alpha$ for $m=0.1 \sim 1.0$

Fig. 5. The influence of lateral contraction coefficient $\alpha$ on values of $\prod \gamma$ and $b_{r}$.

Plot of $b_{r}$ obtained by regression from calculated results is shown in Fig. 5b. It is seen that for all values of $\alpha$ the regression values of $b_{r}$, among a range of 1.98-2.05, are almost consistent to the theoretical value, 2.0. This fact serves as an effective control over the accuracy and a justification of the numerical simulation.

\section{Concluding remarks}

In this paper the power law creep under unidirectional tension by Tabor [19] is formally generalized to the three dimensional case. In order to do this an additional material constant $\alpha$ is introduced to keep track of the effect of lateral contraction. The indentation of such materials using self-similar indenters (conic or pyramidal) is reduced to the indentation of a nonlinear elastic material through dimensional analysis.

Table 4

$\prod_{\gamma}(m=0.1-1.0, \alpha=0.100-0.499)$.

\begin{tabular}{lllllll}
\hline$\alpha$ & $M=0.10$ & $m=0.20$ & $m=0.30$ & $m=0.40$ & $m=0.50$ & $m=1.00$ \\
\hline 0.100 & 2.548 & 1.877 & 1.483 & 1.050 & 0.879 & 0.187 \\
0.250 & 2.584 & 1.878 & 1.500 & 1.074 & 0.903 & 0.188 \\
0.300 & 2.587 & 1.899 & 1.556 & 1.131 & 0.929 & 0.191 \\
0.450 & 2.599 & 1.918 & 1.599 & 1.123 & 0.958 & 0.200 \\
0.490 & 2.653 & 1.950 & 1.586 & 1.133 & 0.945 & 0.201 \\
0.499 & 2.674 & 1.983 & 1.613 & 1.145 & 0.958 & 0.208 \\
\hline
\end{tabular}


The finite element method based on ABAQUS is applied to the numerical simulation of the indentation by conic indenters. The method is shown to yield sufficiently accurate results. Our results show.

The numerical values of $\prod_{\alpha}, \prod_{\beta}$ and $\prod_{\gamma}$ tabulated in Tables $2-4$ can be put back to Eqs. (4) and (5) to yield the values of the load $F(t)$, the projected contact area $A_{c}(t)$ and the hardness $H(t)$ at the given values of $h(t)$.

Although $\prod \gamma$ has been calculated here only for the conic indenter with a half apex angle $70.3^{\circ}$, it can be applied equally well to the standard Berkovich and Vickers indenters, as these indenters have been shown to yield the same loading versus penetration depth relations by Li et al. [22] for work hardening elastic-plastic materials.

Using the results presented here the modern indentation technology may be employed to determine the material constants $b, m$ and $\alpha$ describing the creep property of the class of materials obeying the power law of creep. Because it has been demonstrated [21] that programmed hardness test can yield the value of $b \prod \gamma$ and $m$. With a good estimation of $\alpha$ we know then the value of $\prod \gamma$ from which the value of $b$ then follows. Modern indentation test has the advantage over conventional tensile test in that the former can be easily applied to specimens in the form of thin films, coatings or only available in very small sizes.

\section{Acknowledgements}

The authors would like to thank Prof. Cheng Che-Min for fruitful discussions, and to acknowledge the supports from the National
Natural Science Foundation of China (Grant No. 10772183) and the Intellectual Innovation Project of the Chinese Academy of Sciences (Grant No. KJCX2-YW-L07).

\section{References}

[1] E.H. Lee, Q. Appl. Math. 13 (1955) 183-190.

[2] J.R.M. Radok, Q. Appl. Math. 15 (1957) 198-210.

[3] E.H. Lee, J.R.M. Radok, J. Appl. Mech. 27 (1960) 438-452.

[4] S.C. Hunter, J. Mech. Phys. Solids 8 (1960) 219-234.

[5] G.A.C. Graham, Int. J. Eng. Sci. 3 (1965) 27-46.

[6] G.A.C. Graham, Int. J. Eng. Sci. 5 (1967) 495-514.

[7] W.H. Yang, J. Appl. Mech. 33 (1966) 395-402.

[8] T.C.T. Ting, J. Appl. Mech. 33 (1966) 845-851.

[9] T.C.T. Ting, J. Appl. Mech. 35 (1968) 248-252.

[10] L. Cheng, X. Xia, W. Yu, L.E. Scriven, W.W. Gerberich, J. Polym. Sci.: Part B: Polym. Phys. 38 (2001) 10-22.

[11] P.L. Larrson, S. Carlsson, Polym. Test 17 (1998) 49-75.

[12] S. Shimizu, T. Yanagimoto, M. Sakai, J. Mater. Res. 14 (1999) 4075-4086.

[13] M. Sakai, S. Shimizu, Non-Cryst. Solids 282 (2001) 236-247.

[14] M. Sakai, Philos. Mag. A 82 (2002) 1841-1849.

[15] M.L. Oyen, R.F. Cook, J. Mater. Res. 18 (2003) 139-150.

[16] Y.T. Cheng, C.M. Cheng, Struct. Control Health Monitor. 13 (2006) 561-569.

[17] Y.T. Cheng, C.M. Cheng, Mater. Sci. Eng. A 409 (2005) 93-99.

[18] Y.T. Cheng, C.M. Cheng, J. Mater. Res. 20 (2005) 1046-1053.

[19] T.O. Mulhearn, D. Tabor, J. Inst. Met. 89 (1960) 7-12.

[20] A.P. Bower, N.A. Fleck, A. Needleman, N. Ogbonna, Proc. R. Soc. Lond. Ser. A 441 (1993) 97-124.

[21] Y.T. Cheng, C.M. Cheng, Philos. Mag. Lett. 81 (2001) 9-16.

[22] M. Li, W.M. Chen, N.G. Liang, L.D. Wang, J. Mater. Res. 19 (2004) 73-78. 